\title{
ETTUE COMPARATIVE DE LA TOLẼRANCE AUX BASSES TENSIONS D'OXYGENE CHEZ DEUX PLANAIRES D'EAU DOUCE, DUGESIA TIGRINA ET POLYCELIS FELINA
}

\author{
par Renée Russier-Delolme ${ }^{1}$.
}

\begin{abstract}
Une méthode de détermination du seuil d'oxygène létal en milieu confiné, agité ou stagnant, est mise au point pour deux Planaires d'eau douce, Dugesia tigrina et Polycelis felina.

Bien que voisin de $0,30 \mathrm{mg}$ d'oxygène par litre, ce seuil varie significativement d'une espèce à l'autre, ainsi que sous l'influence de l'agitation.

Aux basses tensions d'oxygène, les deux espèces réduisent considérablement leur métabolisme respiratoire, se comportant done en ajusteurs. L'ajustement est 3 fois plus important chez $D$. tigrina que chez $P$. felina. La première survit également 6 fois plus longtemps dans ces conditions défavorables.
\end{abstract}

\section{A comparative sudy of the tolerance of two freshwater planarians, Dugesia tigrina and Polycelis felina, to low oxygen levels.}

A closed-bottle method was used for determining the lethal level of oxygen for two fresh-water Triclads, Dugesia tigrina and Polycelis felina, in stagnant as well as in stirred water.

The lethal level was found to be about $0,30 \mathrm{mg}$ of dissolved oxygen per litre. However it varies significantly according to the species and to whether the water is stirred or not.

At low oxygen concentrations, both species reduce considerably their metamolism, i. e. they adapt to the low oxygen levels $D$. tigrina does so to a greater extent than $P$. felina. D. tigrina also survives 6 times longer than $P$. felina in such unfavourable conditions.

Dugesia tigrina (Girard) est une Planaire américaine immigrante en Europe. Dans la région lyonnaise, elle a été découverte pour la première fois en décembre 1968 et sa présence a été signalée dans une publication de 1970 par Russier et Lascombe. Elle a été récoltée dans six stations de la région. Elle préfère les eaux stagnantes, chaudes et dans lesquelles la concentration en oxygène peut être assez faible.

Polycelis felina (Dalyell) est une Planaire indigène, montagnarde. Elle occupe des eaux plus courantes, plus froides et dont la teneur en oxygène est plus élevée et plus stable.

1. Equipe d'Ecophysiologie - Département de Biologie et Zoologie, Université Claude-Bernard, Lyon I, 43, boulevard du 11-Novembre-1918, 69 - Villeurbanne. 
Une méthode de détermination du seuil létal d'oxygène est mise au point, de façon à pouvoir comparer la tolérance aux basses tensions chez ces deux Planaires et montrer si leur tolérance à ce facteur contribue à déterminer le type d'habitat des animaux.

Les exemplaires des deux espèces proviennent des milieux suivants :

- D. tigrina : étang des Bruyères sur le ruisseau de Méginand, altitude $240 \mathrm{~m}$, précédemment décrit (Russier et Lascombe, 1970 ).

- P. felina : ruisselets des sources de l'Yzeron, petite rivière des Monts du Lyonnais, altitude de la station : $800 \mathrm{~m}$ (Fiasson, 1964).

\section{1. - Méthodes de détermination du taux d'oxygène létal.}

\section{1. - Travaux antérieurs.}

Comme pour la température, il existe, pour l'oxygène, deux méthodes de mesure du seuil létal : l'une à taux d'oxygène constant et l'autre à taux variable.

Dans la première on mesure le temps de survic aux différentes tensions ; la tension létale est définie comme celle à laquelle $50 \%$ des sujets survivent pendant un temps donné, 24 heures en général (Sprague, 1963). Shepard (1955), quant à lui, fait intervenir un temps de 5 minutes seulement. C'est une méthode assez rigoureuse mais elle nécessite un appareillage permettant de maintenir la stabilité de la concentration en oxygène dans le flacon où respirent les animaux.

La seconde méthode consiste à enfermer les animaux à étudier dans des flacons contenant de l'eau appauvrie en oxygène et à mesurer le taux de ce gaz au moment de leur décès. Elle a été utilisée, par exemple, par Abbott (1960) pour les Planaires, Burbanck, Edwards et Burbanck (1948), Park, Gregg et Lutherman (1940) pour l'Ecrevisse. Ces auteurs mesurent le taux d'oxygène, par la méthode de Winkler, après la mort du dernier animal.

\subsection{Mise au point d'une méthode a taux d'oxygène variable.}

a) Principe de la méthode.

Elle consiste à mesurer le taux d'oxygène pour différents pourcentages de mortalité. La variation de l'un en fonction de l'autre est alors exprimée graphiquement par la droite de régression, que l'on considère comme décrivant approximativement le phénomène, étant donné la variabilité des mesures. Le taux létal est alors défini 
comme le taux d'oxygène qui, d'après la droite de régression, correspond à $50 \%$ de décès.

b) Mode opératoire.

Après leur récolte, les animaux sont mis en élevage à $15{ }^{\circ} \mathrm{C}$. Les expériences étant faites à $20^{\circ} \mathrm{C}$, ils sont ensuite acclimatés, à cette température pendant 48 à 72 heures en les laissant à jeûn, puis introduits par groupes d'une dizaine dans des flacons de verre jaugés, à fermeture émeri, immergés dans un bain-marie. L'eau contenue dans les flacons est de l'eau de la canalisation urbaine déchlorée et appauvrie en oxygène par barbotage d'azote jusqu'à ce qu'elle contienne 0,6 à $1 \mathrm{mg} / \mathrm{l}$ d'oxygène. Le dosage de l'oxygène est fait par la méthode polarographique à l'aide de l'analyseur Beckman Fieldlab, dans lequel l'électrode est protégée par une membrane de téflon. La compensation de température se fait par réglage manuel. Cette méthode de mesure de l'oxygène est rapide et fidèle, une fois effectué l'étalonnage du point 0 et du point de saturation (Nösel, 1969). La précision est de l'ordre de 1/2 graduation de l'appareil, soit $\pm 0,05 \mathrm{mg} / \mathrm{l}$.

Après un certain intervalle de temps, que l'on note chaque fois, les animaux commencent à mourir. La tension d'oxygène est alors mesurée à nouveau dans les flacons et les survivants sont transvasés dans de l'eau aérée, à la température de l'expérience, c'est-àdire $20^{\circ} \mathrm{C}$. Le nombre de décès est noté au bout de 24 heures. On ne tient compte que des flacons comportant à la fois des morts et des survivants.

Pour chacune des deux espèces, l'expérience est effectuéc aussi bien en eau stagnante qu'en eau agitéc. Deux facteurs interviennent donc: l'espèce et l'agitation. Celte dernière est obtenue à l'aide d'un barreau aimanté dont la taille et la vitesse de rotation sont réglées de façon à ne pas blesser les animaux. Ceux-ci s'immobilisent sur les parois verticales du flacon et ne sont que rarement pris dans le mouvement tourbillonnaire.

\section{Le seuil létal chez D. tigrina et $P$. felina.}

\section{1. - RÉsultats.}

Pour chaque espèce en eau stagnante et en eau agitée, 23 mesures de la tension finale d'oxygène ont été effectuées, correspondant à divers pourcentages de décès. Les expériences fournissent quatre droites dont la figure montre un exemple. L'équation de régression de ces droites permet alors de calculer le taux d'oxygène létal, défini comme le taux entraînant $50 \%$ de mortalité, Les résultats sont rassemblés dans le tableau I. 
Il pourrait sembler plus simple d'obtenir directement le taux létal en procédant au dosage de l'oxygène dans les flacons après la mort de 5 animaux sur 10 , mais le nombre de morts, à un instant donné, est pratiquement impossible à évaluer pendant l'expérience. Ce nombre n'est déterminé de façon exacte qu'après séjour de 24 heures en eau aérée.

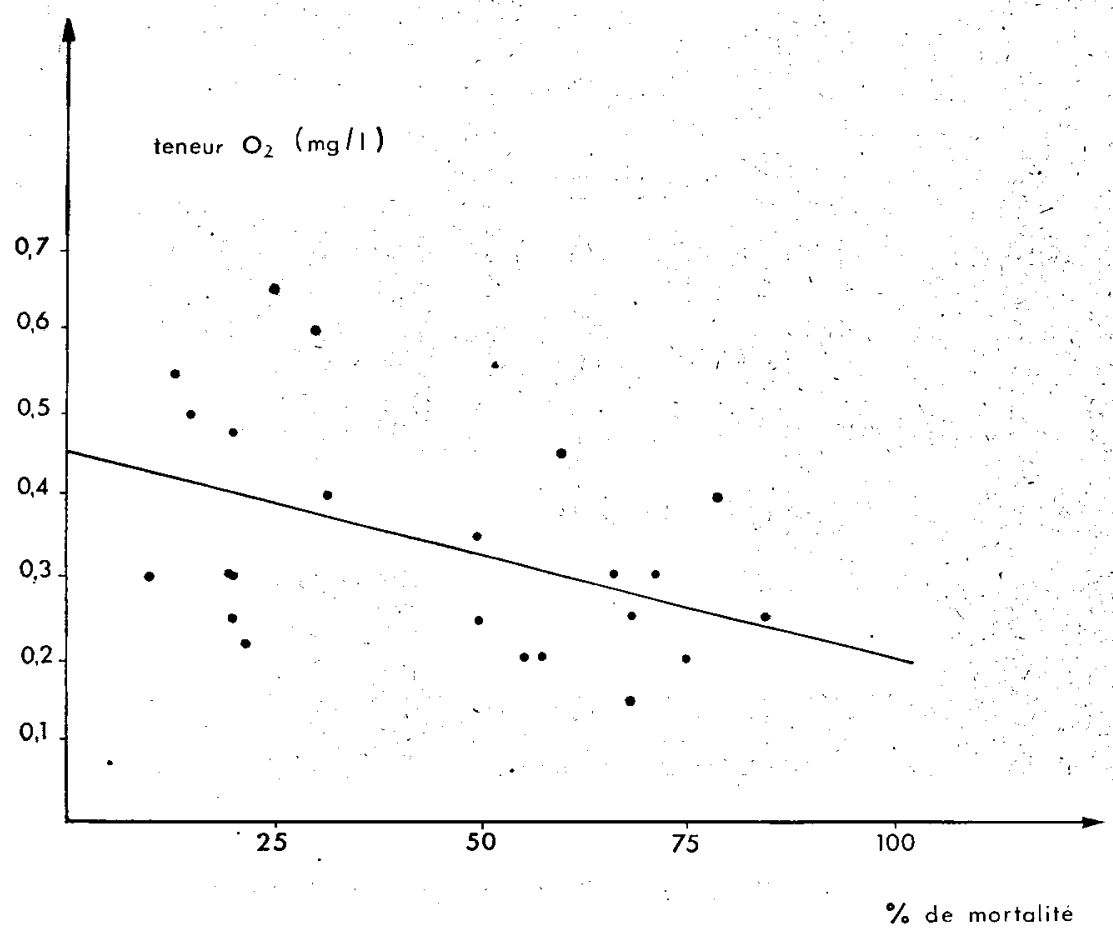

Fig. 1. - Relation entre le pourcentage de mortalité et la tension d'oxygène finale chez Dugesia tigrina en milieu stagnant.

Les résultats apparaissent assez voisins les uns des autres. Les tensions sont très basses si on les compare à celles obtenues chez d'autres invertébrés aquatiques. Madsen (1968) mesure pour deux larves de Plécoptères la concentration létale en oxygène, à $10^{\circ} \mathrm{C}$. En milieu stagnant, elle est égale à $5 \mathrm{mg} / \mathrm{l}$ pour l'espèce d'eau courante et à $1 \mathrm{mg} / \mathrm{l}$ pour celle d'eau calme. Cette tension létale diminue quand l'eau est agitée, tombant, pour les deux espèces, respectivement à $2 \mathrm{mg} / \mathrm{l}$ et $0,5 \mathrm{mg} / \mathrm{l}$. Pour des Crustacés, à $20^{\circ} \mathrm{C}$, Sprague (1963) obtient $50 \%$ de mortalité en 24 heures à $2,2 \mathrm{mg} / \mathrm{l}$ d'oxygène chez Gammarus pseudolimnaeus, à $4,3 \mathrm{mg} / 1$ chez $G$. 
fasciatus et à $0,03 \mathrm{mg} / \mathrm{l}$ chez Asellus intermedius. Nos résultats rejoignent ceux de Hyman (1929), citée par Abbott en 1960, qui indique que plusieurs espèces de Planaires peuvent vivre dans des teneurs en oxygène de $0,6 \mathrm{mg} / \mathrm{l}$ et sont plus ou moins indépendantes de ce facteur.

Tableau I. - Tension d'oxygène létale pour $D$. tigrina et $\boldsymbol{P}$. felina en eau stagnante et agitée.

- Première partie du tableau : détermination des quatre tensions létales. L'équation de la droite de régression exprime les variations de la tension d'oxygène finale (en $\mathrm{mg} / \mathrm{l}$ ) en fonction du pourcentage de mortalité. La tension létale est celle qui correspond à $50 \%$ de mortalité.

- Deuxième partie du tableau : comparaison des quatre tensions létales. Analyse de covariance, pour l'ensemble des données. effectuée selon la méthode des $\mathrm{Y}$ réduits (Lison 1958).

Les valeurs critiques de $F$, pour le nombre de degrés de liberté (D. L.) indiqué et le niveaux de probabilité de $5 \%$ et $1 \%$ sont respectivement de 3,96 et de 6,96 .

* la valeur de $\mathrm{F}$ dépasse le niveau de $5 \%$ (différence significative).

* Ia valeur de $\mathrm{F}$ dépasse le niveau de $1 \%$ (différence hautement significative).

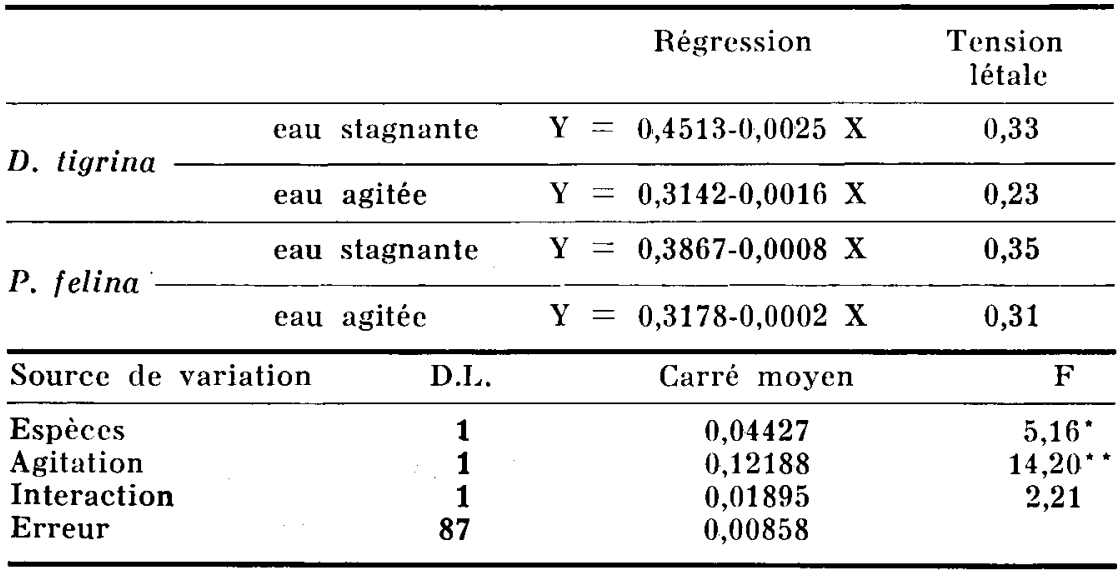

\section{2. - INTERPRÉTATION DES RÉSULTATS : COMPARAISON ENTRE ESPÈCES} ET INFLUENCE DE L'AGITATION.

L'existence d'une relation inverse entre la tension d'oxygène finale et le taux de mortalité était implicite dans la définition du taux létal à $50 \%$ des individus. Elle a été démontrée, pour l'ensemble des données, en décomposant la variation totale en variation due à la régression de la tension finale sur le pourcentage de mortalité et déviations depuis cette régression. Ainsi que le montre Lison (1958), les déviations depuis la régression représentent les variations non contrôlées, c'est-à-dire l'erreur, et peuvent donc être utilisées pour tester la signification statistique de la régression. La valcur critique de la distribution de $F$ pour le niveau de probabilité de 
$1 \%$ est de 6,96 . La valeur obtenue ici, $\mathrm{F}=11,24$, est bien supérieure : la variation due à la régression dépasse donc nettement le seuil de signification. C'est dire que lorsqu'on considère les résultats globalement, tous les sujets ne meurent effectivement pas à la même tension, mais que cette dernière diminue progressivement entre la mort du premier et celle du dernier animal de chaque lot.

Ainsi, pour tester statistiquement l'influence des deux facteurs, espèce et agitation, sur la tension finale, il sera utile de tenir compte du troisième facteur important : le taux de mortalité. Or, l'influence de ce dernier peut être éliminée si elle est linéaire, ou du moins fortement réduite si elle ne l'est pas, grâce à l'analyse de covariance, qui consiste, en définitive, selon Lison, à comparer les tensions finales comme si elles avaient été mesurées à un même pourcentage de mortalité, $50 \%$ par exemple. L'analyse permettra donc de comparer statistiquement les quatre valeurs de tension létale du tableau I (première partie).

La deuxième partie de ce même tableau résume cette analyse. L'interaction n'est pas statistiquement significative; l'influence de l'agitation doit être considérée comme semblable sur les deux espèces. Par contre, ces deux espèces perdent $50 \%$ de leurs effectifs à des tensions qui en moyenne diffèrent significativement l'une de l'autre : le taux létal d'oxygène dissous est plus bas chez $D$. tigrina que chez $P$. felina, aussi bien en eau stagnante qu'en eau courante. En outre, ce taux est diminué de façon hautement significative par l'agitation de l'eau qui favorise donc la survie en atténuant l'effet du manque d'oxygène.

Ce résultat est à mettre en parallèle avec celui de Madsen (1968). Chez les Planaires, comme chez les larves de Plécoptères, l'espèce vivant en eau stagnante (en l'occurrence $D$. tigrina) survit à un taux d'oxygène plus faible que l'espèce d'eau courante et dans les deux cas le niveau létal est réduit par l'agitation. Le milieu agité fait preuve, selon Madsen, d'une meilleure «disponibilité en oxygène ».

Mais, chez nos Planaires, les différences dans les tensions létales ont été mises en évidence grâce à un test statistique assez précis : l'analyse de covariance. Elles n'atteignent que le dixième de mg, valeur bien inférieure à ce que trouve Madsen chez les Plécoptères.

\section{3. - Comparaison des temps de survie aux basses tensions.}

Au cours de l'expérience ont été mesurés les temps de survie de chaque lot d'animaux. Les résultats concernant la moyenne, pour 23 mesures, des durées de survic, exprimées en heures, figurent au tableau II (première partie). 
Tableau II. - Durée moyenne de survic à faible concentration d'oxygène chez D. tigrina et $P$. felina.

- Première partie du tableau : moyenne des temps de survie, exprimés en heures, de chaque lot d'animaux, en milieu stagnant et en milieu agité. - Deuxième partie du tableau : analyse de variance des données. Les valeurs critiques de $F$, pour le nombre de degrés de liberté (D. L.) indiqué et les niveaux de probabilité de $5 \%$ et $1 \%$ sont respectivement 3,96 et 6,96 .

\begin{tabular}{|c|c|c|c|}
\hline \multicolumn{2}{|l|}{ D. tigrina } & \multicolumn{2}{|c|}{ P. felina } \\
\hline Eau stagnante & Eau agitée & Eau stagnante & Eau agitée \\
\hline 32,45 & 36,12 & 5,72 & 6,15 \\
\hline Source de variation & D.L. & Carré moyen & $\bar{F}$ \\
\hline Espèces & 1 & 18473,66 & $207,13^{*}$ \\
\hline Agitation & 1 & 92,51 & 1,03 \\
\hline Interaction & 1 & 64,35 & $<1$ \\
\hline Erreur & 88 & 89,19 & \\
\hline
\end{tabular}

Pour connaître l'influence des deux facteurs espèce et agitation, ainsi que de leur interaction, sur la durée de survic des animaux, il suffit de traiter les résultats par l'analyse de variance (deuxième partie du tableau II). L'expérience est du type $2 \times 2$, les deux facteurs agissant chacun à deux niveaux différents.

Aux basses tensions d'oxygène, l'agitation ne prolonge pas de façon sensible la survie des animaux. Par contre, suivant que l'on a affaire à l'une ou l'autre espèce, cette durée de survie varie de façon hautement significative. Dans ces conditions défavorables, $D$. tigrina résiste donc plus longtemps que $P$. felina (environ 6 fois plus selon le tableau II, $1^{\text {re }}$ partie). Puisque dans ces expériences la durée de survie de $D$. tigrina est bien supérieure à celle de $P$. felina, il serait intéressant de voir si cette différence s'accompagne d'une variation importante dans la consommation horaire d'oxygène de ces deux espèces.

\section{4. -- Consommation d'oxygène en milieu stagnant.}

Les expériences précédentes, où l'on connaît les teneurs en oxygène initiales et finales, ainsi que les durées de séjour des animaux, permettent de calculer la consommation horaire d'oxygène de ces derniers, à condition de ne tenir compte que des flacons dans lesquels le pourcentage de mortalité est très faible. En effet, la mort d'un animal ne signifie pas nécessairement que sa consommation d'oxygène cesse : Vollenweider et Ravera (1958) notent que, par par suite de la respiration des bactéries se développant sur le cadavre, la consommation d'oxygène résultant de la présence d'une Daphnie morte est supérieure à celle d'une Daphnie vivante. 
Par comparaison, d'autres expériences de consommation d'oxygène ont été effectuées à saturation, en eau stagnante à $20^{\circ} \mathrm{C}$, simultanément chez les deux espèces de Planaires. La méthode utilisée est très proche de celle employée précédemement. Les animaux, à jeun, acclimatés pendant 48 à 72 heures à la température de $20{ }^{\circ} \mathrm{C}$, sont enfermés par groupes de dix dans les flacons. Les mesures ont donc lieu en milieu confiné avec dosage de l'oxygène dissous, au moyen de l'analyseur polarographique, dans les flacons d'expérience avant et après respiration des animaux, ainsi que dans des flacons témoins.

Les différences entre les deux séries d'expériences portent sur la tension d'oxygène au départ, proche de la saturation $(8,8 \mathrm{mg} / \mathrm{l}$ à $\left.20^{\circ} \mathrm{C}\right)$ dans l'une et faible $(0,6$ à $1 \mathrm{mg} / \mathrm{l})$ dans l'autre. En fin d'expérience la teneur en oxygène est voisine de $5 \mathrm{mg} / \mathrm{l}$ dans la première série, de $0,35 \mathrm{mg} / \mathrm{l}$ dans la deuxième. La consommation est exprimée en $\mu \mathrm{g}\left(=10^{-3} \mathrm{mg}\right)$ par heure et par animal. Comme les précédentes, l'expérience comporte deux facteurs, l'espèce et la tension d'oxygène au départ, agissant chacun à deux niveaux différents. Les résultats, correspondant chacun à la moyenne de 15 mesures, sont les suivants pour $D$, tigrina et $P$. felina respectivement : à saturation 20,22 et $29,95 \mu \mathrm{g}$ d'O $\mathrm{O}_{12}$ par animal et par heure, à basse tension 0,58 et $2,94 \mu \mathrm{g} \mathrm{d} \mathrm{d}_{2}$ par animal et par heure. L'analyse de variance permetlra d'évaluer le rôle de chacun des facteurs ainsi que celui de leur interaction, c'est-à-dire de leur influence réciproque.

Ces résultats montrent qu'à tension basse il apparaît dans les deux cas une réduction importante de la consommation. Mais cette consommation par individu varie d'une espèce à l'autre. L'analyse des données de ce tableau fournirait uniquement la comparaison des valeurs absolues et de leurs différences. Ceci n'a pas d'intérêt pour nous ; ce qu'il convient d'étudier c'est de combien de fois la consommation est réduite, de part et d'autre, sous l'influence des basses tensions, c'est-à-dire l'abaissement relatif de cette consommation. On atteint ce but en pratiquant l'analyse sur les données auxquelles on a fait subir la transformation logarithmique (tableau III, première partie). La différence des données logarithmiques représente alors le rapport des données brutes.

Le test F (tableau III, deuxième partie), montre que la variation due à l'interaction est significative. Les deux espèces réagissent différemment l'une de l'autre. Selon Lison (1958), il est done nécessaire d'effectuer un test $t$ portant sur la différence entre les moyennes logarithmiques prises deux à deux. Comme le montre la première partie du tableau III, toutes les différences sont supé-

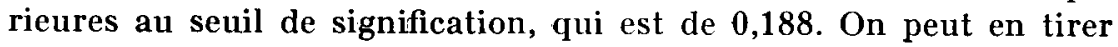
les conclusions suivantes : 
Tableau III. - Consommation d'oxygène à saturation et à basse tension, chez $P$. felina et $D$. tigrina en milieu stagnant.

- Première partie du tableau : comparaison des quatre consommations. Les valeurs représentent les moyennes des logarithmes des données et leurs différences. Ces différences sont égales au logarithme du rapport entre les données brutes, rapport qui figure entre parenthèses. Selon Lison (1958) la plus petite différence significative, pour 56 degrés de liberté, est égale à 0,188 pour le seuil de probabilité de $5 \%$ et 0,250 pour le seuil de probabilité de $1 \%$.

- Deuxième partie du tableau : influence des deux facteurs espèce et tension d'oxygène sur la consommation d'oxygène. Analyse de variance des données de la première partie du tableau. Valeur critique de $F$ au niveau de $1 \%: 7,12$.

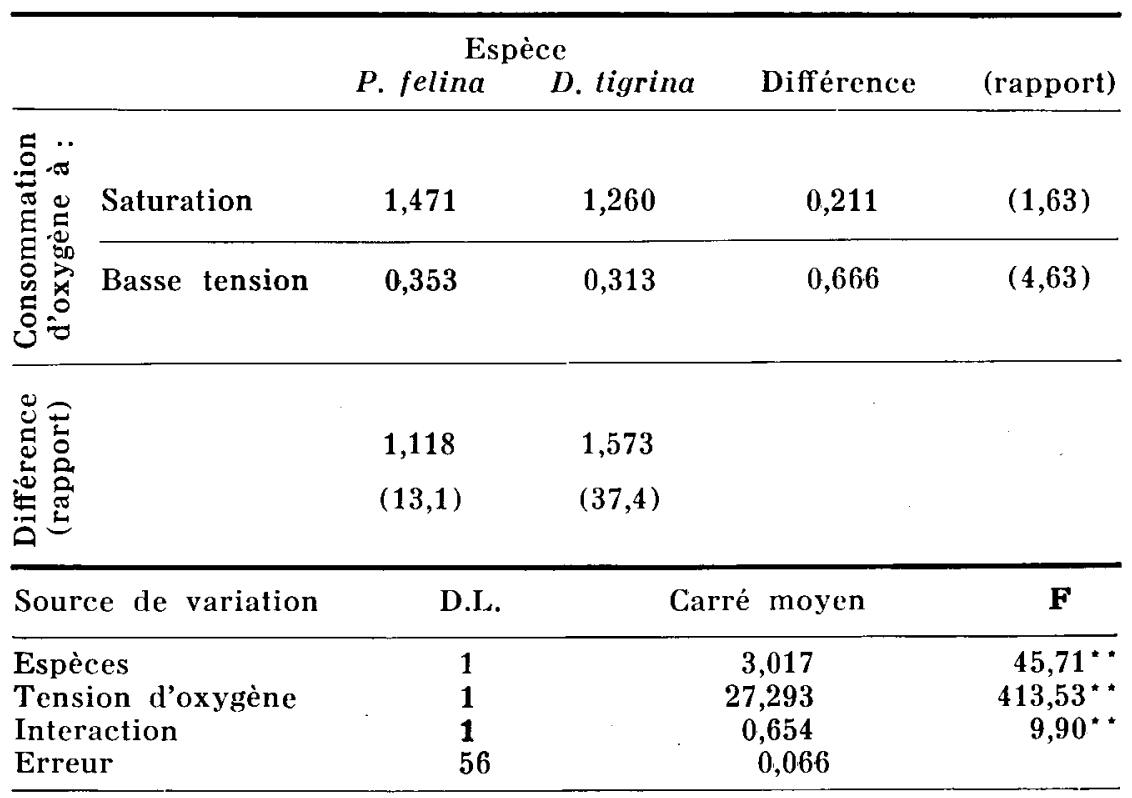

- Un individu de $P$. felina consomme toujours plus d'oxygène qu'un individu de $D$. tigrina, aussi bien à saturation qu'aux basses tensions. Or, nous avons constaté aussi bien en milieu stagnant qu'en milieu agité, une différence dans le comportement des deux espèces. Par rapport à celle des $D$. tigrina, l'activité des $P$. felina, surtout en début d'expérience, est plus intense. Elles se déplacent davantage dans le flacon. Cette activité plus grande chez $P$. felina est peut-être à mettre en relation avec sa consommation d'oxygène plus élevée.

- L'abaissement de la teneur en oxygène réduit la consommation aussi bien chez $D$. tigrina que chez $P$. felina, qui se conduisent donc en ajusteurs. L'ajustement est, selon Prosser (1955), la possibilité d'adapter sa consommation d'oxygène à la quantité de ce gaz présente dans le milieu. 
- Mais surtout, la valeur de l'interaction montre que l'ajustement est nettement plus important chez $D$. tigrina que chez $P$. felina. L'influence de la tension en oxygène dissous fait varier la consommation dans un rapport de 1 à 37 chez la première et seulemen! de 1 à 13 chez la seconde. La faculté d'ajustement de la première représente environ 3 fois celle de la seconde.

\section{5. - Discussion et conclusions.}

Cette méthode de détermination du scuil létal d'oxygène dissous applicable à d'autres représentants de la faune, nous a permis de comparer les capacités de deux espèces de Planaires, D. tigrina et $P$. felina. Elles montrent une très grande tolérance vis-à-vis du facteur oxygène dissous et se comportent en ajusteurs mais à des degrés variables pour l'une et l'autre.

Les résultats sont à rapprocher de ceux obtenus pour des Ecrevisses :

- Park, Gregg et Lutherman (1940) ont testé la lolérance d'Écrevisses d'eau calme et d'eau courante aux basses tensions d'oxygène par détermination de la concentration létale en milieu confiné. Ils constatent que les animaux d'eau courante meurent plus rapidement et à un taux d'oxygène plus élevé que ceux d'eau calme. Il en est de même ici, l'espèce d'eau stagnante, D. tigrina, survit plus longtemps et tolère des tensions d'oxygène légèrement plus basses que l'espèce d'eau courante, $P$. felina.

- Burbanck, Edwards et Burbanck (1948) en utilisant une méthode identique établissent une comparaison entre des Ecrevisses cavernicoles et des Écrevisses épigées d'eau courante. Les deux espèces réduisent la tension d'oxygène de la même valeur mais les Ecrevisses cavernicoles survivent plus longtemps. Elles ne tolèrent pas des tensions d'oxygène plus basses que les Ecrevisses épigées, mais elles ont un métabolisme plus faible qui réduit la tension d'oxygène plus lentement. D. tigrina se comporte comme les Ecrevisses cavernicoles, son métabolisme respiratoire étant plus faible que celui de $P$. felina, elle survit plus longtemps.

D. tigrina se montre mieux adaptée aux caux stagnantes susceptibles de subir de plus grandes variations de concentration en oxygène que le cours supérieur des ruisseaux habité par $P$. felina. Résistant aux basses tensions plus de 30 heures à $20^{\circ} \mathrm{C}, D$. tigrina paraît, en particulier, pouvoir subir impunément l'abaissement nocturne de la concentration qui se produit dans les milieux de petites dimensions encombrés de végétation, tels que celui décrit par Zaaijer et Wolvekamp (1958).

La détermination du seuil létal d'oxygène dissous semble être d'une grande importance dans le cadre d'études sur la pollution. La 
moyenne de $0,3 \mathrm{mg} / \mathrm{l}$ mesurée ici correspond à une teneur très basse dans le milieu. De ce point de vue, nos deux espèces ne peuvent donc avoir qu'une faible valeur indicatrice : la présence de $P$. felina indique pratiquement que l'oxygène ne disparaît jamais dù milieu, celle de $D$. tigrina qu'il ne disparaît jamais plus de quelques heures.

Cependant, se fondant sur l'étude de Seibold (1955), Besch (1967) considère qu'aucune Planaire ne résiste à une charge moyenne en substances toxiques. Certaines d'entre elles, parmi lesquelles il cite Polycelis, seraient même très sensibles à ce genre de pollution. Nos conclusions viennent donc étayer celles de cet auteur lorsqu'il considère les Planaires comme des indicateurs spécifiques non de l'oxygène dissous, mais de la charge en ions toxiques.

\section{TRAVAUX GITÉS}

Aввotr (B. J.). 1960. - A note on the oxygen and temperature tolerances of the Triclads Phagocata gracilis (Haldeman)) and Dugesia tigrina (Girard). Va. J. Sci. n. s., $11: 1-8$.

Besch (W.). 1967. - Biologischer Zustand und Abwasserbelastung der Fliessgewässer Südwürttembergs. Veröff. Landesst. Natursch. Landsch. Baden-Würtlemberg, 35: 111-128.

Burbanck (W. D.), Eowards (J. P.) et Burbanck (M. P.). 1948. -- Toleration of lowered oxygen tension by cave and stream crayfish. Ecology, 29 : 360-367.

Fissson (S.). 1964. - Recherches écologiques sur la faune d'une rivière de la région lyonnaise : l'Yzeron. Thèse Sc., Lyon. $182 \mathrm{p}$.

Lison (L.). 1958. - Statistique appliquée à la biologie expérimentale. 346 p. Paris.

MAdSEN (B. L.). 1968. - The distribution of nymphs of Brachyptera risi Mort. and Nemoura flexuosa Aub. (Plecoptera) in relation to oxygen. Oikos, $19: 304-310$.

Nöseı. (H.). 1969. - Kritische Untersuchungen über die Genauigkeit und Zuverlässigkeit membran-polarometrischer $\mathrm{O}_{\mathrm{z}}$-Messungen in der Abwasserpraxis. Wasserwirtschaft, Sluttg., $59: 1-8$.

Park (T.), GregG (R. E.) et LuthermaN (C. Z.), 1940. - Toleration experiments by ecology classes. Ecology, 21 : 109-111.

Prosser (C. L.) . 1955. - Physiological variation in animals. Biol. Rev., $30: 229-262$.

Russier (R.) et Lascombe (C.). 1970. - La Planaire américaine Dugesia tigrina dans la région lyonnaise : écologie et tolérance thermique. Bull. mens. Soc. linn, Lyon, $39: 197-206$. 
SEIBold (A.). 1955. - Die Einwirkung von organischen Fäulnisstoffen auf tierische Leitformen des Saprobiensystems. Vom Wass., 22 : 90-166.

Shepard (M.P.). 1955. - Resistance and tolerance of youg speckled trout (Salvelinus fontinalis) to oxygen lack, with special reference to low oxygen acclimation. J. Fish. Res. Bd Can., 12 : 587-605.

Sprague (J. B.). 1963. - Resistance of four freshwater Crustaceans to lethal high temperature and low oxygen. J. Fish. Res. Bd. Can., $20: 387-415$.

Vollenweider (R. A.) et Ravera (O.). 1958. - Preliminary observations on the oxygen uptake by some freswater zooplancton. Verh. int. Ver. Limnol., 13 : 369-380.

ZaAiJer (J.J.P.) et Wolvekamp (H. P.). 1958. - Some experiments on the haemoglobin-oxygen equilibrium in the blood of the ramshorn (Planorbis corneus L.). Acta physiol. pharm. neerl., 7 :56-77. 